\title{
A comment on "Regular structures of the solar photosphere"
}

\author{
M. P. Rast \\ High Altitude Observatory, National Center for Atmospheric Research ${ }^{\star}$, PO Box 3000, Boulder, CO 80307, USA \\ Received 11 July 2002 / Accepted 23 July 2002

\begin{abstract}
A recent Letter to the Editor (Getling \& Brandt 2002) suggests that solar granulation is not entirely random, instead showing large scale spatial and long term temporal coherence. The authors cite as evidence the persistence of bright granular size objects in images even after long term temporal averaging, the reoccurrence of bright granules in time series at locations of local maxima in the average image, and the presence of large scale regular structures in time-average images. This paper demonstrates that all three of these observations are consistent with a completely random and changing flow pattern and do not require self organization of the granular flows.
\end{abstract}

Key words. solar photosphere - granulation - convection patterns

\section{Introduction}

In a recent Letter to the Editor, Getling \& Brandt (2002) argue for a previously unknown self-organization of granular flows, based on the persistence of granular scales and the emergence of larger scale patterns in time-averages of a high-quality eighthour image sequence. We show here that their observations are instead consistent with a completely random and changing flow pattern of typical granular lifetime.

\section{Artificial data}

Considerable care was taken to construct a series of random images evolving on a characteristic timescale and containing no hidden spatial or temporal correlations. Each image is the additive superposition of $192^{2}$ two-dimensional Gaussians. Four properties, the amplitude, full width at half maximum, $x$ center coordinate, and $y$ center coordinate of the individual Gaussians are assigned random values at intervals normally distributed about a mean lifetime. All four of the properties, as well as the time interval between the assigned values, are randomly selected independently and out of phase with one another. The lifetime intervals are normally distributed about a mean value of 5 min with a standard deviation of one-half; amplitudes are normally distributed about zero with a standard deviation of one; full widths at half maximum are normally distributed about a value of five pixels with a standard deviation of onehalf; and $x$ and $y$ coordinates random walk (from an initial position near grid points) with a step size normally distributed about zero pixels per mean lifetime with a standard deviation of 1.25. The lifetime distribution is truncated to lie between zero and ten minutes, and the full widths at half maxima are forced to be greater than zero and less than ten pixels. The Gaussian

\footnotetext{
* NCAR is sponsored by the National Science Foundation.
}

amplitudes and random walk step size distributions are unconstrained. Between the randomly assigned properties characterizing the Gaussians after each lifetime, cubic spline interpolation yields values at $20 \mathrm{~s}$ intervals, so that each property evolves smoothly and independently from one random value to the next with a characteristic lifetime centered around $5 \mathrm{~min}$. Finally, all $192^{2}$ Gaussians are additively superimposed at each $20 \mathrm{~s}$ time step to yield 1486 images of a randomly evolving intensity pattern of 8.25 hours duration. A single such image at $192 \times 192$ spatial resolution is displayed in Fig. 1a. Note that the image series so constructed is meant to contain no physics. It is not an attempt to model solar granulation, and is used only to show that some properties of the solar granulation are consistent with a strictly random field evolving with a characteristic lifetime.

\section{Results}

Using this artificially produced series of images we note the following three items (as did Getling \& Brandt 2002 for real granulation images):

1. Averaging the entire time series together produces an average image which is still mottled on the scale of any chosen individual image (Fig. 1c). This does not demonstrate persistence of the pattern over times much longer than the mean lifetime. It can instead be explained as follows. The spatial spectrum of a random superposition of two dimensional objects behaves as

$|\tilde{I}| \propto \frac{k}{\sqrt{N}}|\tilde{S}|$

where $k$ is the horizontal wavenumber, $N$ is the number of individual objects superimposed, and $\tilde{S}$ is the spectrum of each of these. The effect of adding more images, with increasing averaging time, is to add more randomly placed objects, and the shape of the spectrum (including location of maximum power) 
(a)

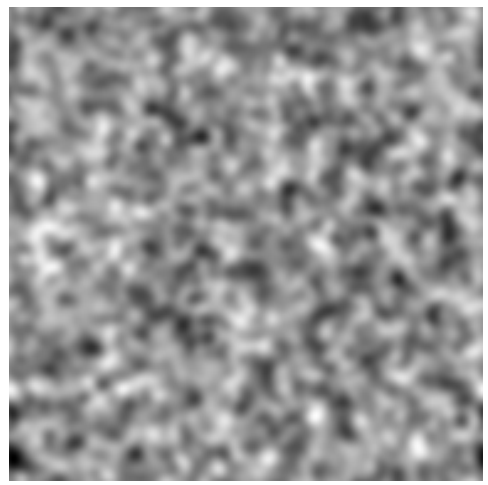

(b)

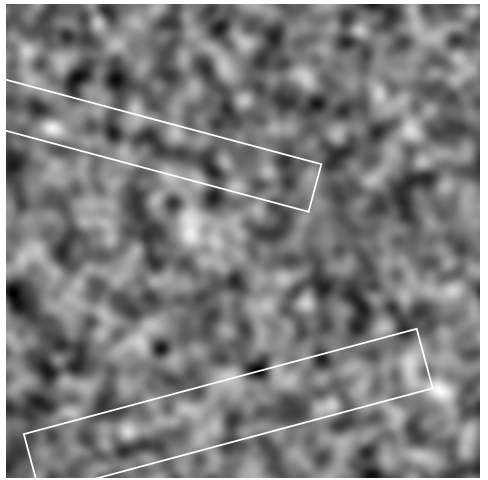

(c)

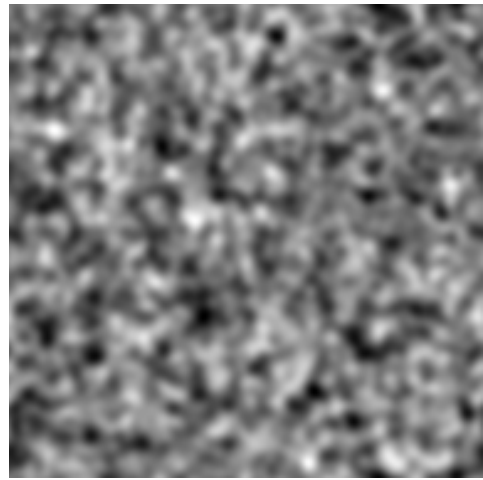

Fig. 1. In a), a single image from the middle of the 2.5 hour interval of the randomly generated intensity time series shown averaged in b). In c), the random intensity pattern averaged over the entire 8.25 hour time-span of the artificial data set.

remains unchanged (Fig. 2b). The amplitude of the spectrum, or root-mean-square contrast of the average images (Fig. 2a), smoothly decreases. Note, however, that the image contrast remains quite high even when averging over the full 8.25 hours, and that its dependence on averaging time is nearly identical to that plotted by Getling \& Brandt (2002) for real solar granulation. The reduction in image contrast is substantially slower than $1 / \sqrt{N_{\mathrm{i}}}$, where $N_{\mathrm{i}}$ is the number of images. It also appears somewhat slower than $1 / \sqrt{N_{1}}\left(N_{1}=1+T / \tau\right.$ with $T$ the averaging time and $\tau$ the mean lifetime of the objects making up the images), a measure of the number of independent images in the series. On closer inspection the difference between the measured reduction and $1 / \sqrt{N_{1}}$ is largely due to an offset introduced at short averaging times (see inset in Fig. 2a). This is not an artifact of the initial random state (the same curve is obtained no matter where in the image series the averaging is initiated), but a real effect due to the fact that the objects making up the image evolve out of phase, and so it takes approximately one mean lifetime for the root-mean-square fluctuations to begin to drop as $1 / \sqrt{N_{1}}$. Real images of solar granulation obey the same statistics. Of the many images taken over a limited period of time only a relatively small number sample an independent state because of the finite lifetime of the flow pattern. Even if the mean granular lifetime were as short as 5-10 min, as in our artificial data, the root-mean-square fluctuations would decay at a rate consistent with that measured by Getling \& Brandt (2002).

2. If a point of local maximum is selected from an average image, the time variation of the intensity at that point shows series of well defined peaks with a period of repetition on the order of the granular life time (Fig. 2c). This is because the bright locations in the average image are those at which the time series happened to contain bright "events" in successive random images. That is why they are bright in the average image. Likewise, locations which are local minima in the average image show repeated dark "events". This is statistical, not dynamical, reoccurrence. The anticorrelation between neighboring bright and dark events discussed by Getling \& Brandt (2002) for the granulation time series occurs only infrequently in our random images (see $t=270-310$ in Fig. 2c). It likely occurs more often for randomly located convective elements because of the dynamical link between upflows and downflows. If the granulation time series by chance contains a series of bright granules at one location (and thus the time-average image appears locally bright there) then a neighboring location is likely to have repeated convective downflows and appear dark. This is an indication of spatial correlation in a convective flow without necessarily dynamical temporal reoccurrence.

3. Finally, the time-average images tend to show more coherent large scale regular structures ("ridges or trenches", light and dark features being equivalent in these randomly generated fields) than do individual images (Fig. 1). This is particularly true for intermediate averaging times (Fig. 1b, chosen to match the averaging time suggested by Getling \& Brandt 2002, not for the structures it contains), with the structures becoming less pronounced for longer averaging intervals. This is likely due to the relatively small number statistics over these short times, so that accidental chains of individual like sign objects appear as linear features (Maunder 1894). Additionally, it may be that dark artifacts of this nature are more common in averages of real granulation images than they are here because of the connectivity of the intergranular lanes in the original single images (not present in this artificial data) and the smoothing of these upon averaging. Note that the average granulation images of Getling \& Brandt (2002) look very much like the randomly generated intensity patterns in this paper, whereas single snapshots of granulation appear quite different, showing sharper intergranular lanes.

\section{Conclusion}

An artificially constructed time series of random images can reproduce all the main features reported by Getling \& Brandt (2002) for real granulation images. Their results are statistical properties of a random field and not due to dynamical self-organization or repetition in the underlying granular flow. Since granulation evolves with a characteristic lifetime, it is the number of lifetimes spanned in the time series, not the total number of images, which dominates the statistics. The root-mean-square fluctuations reported for averages over 

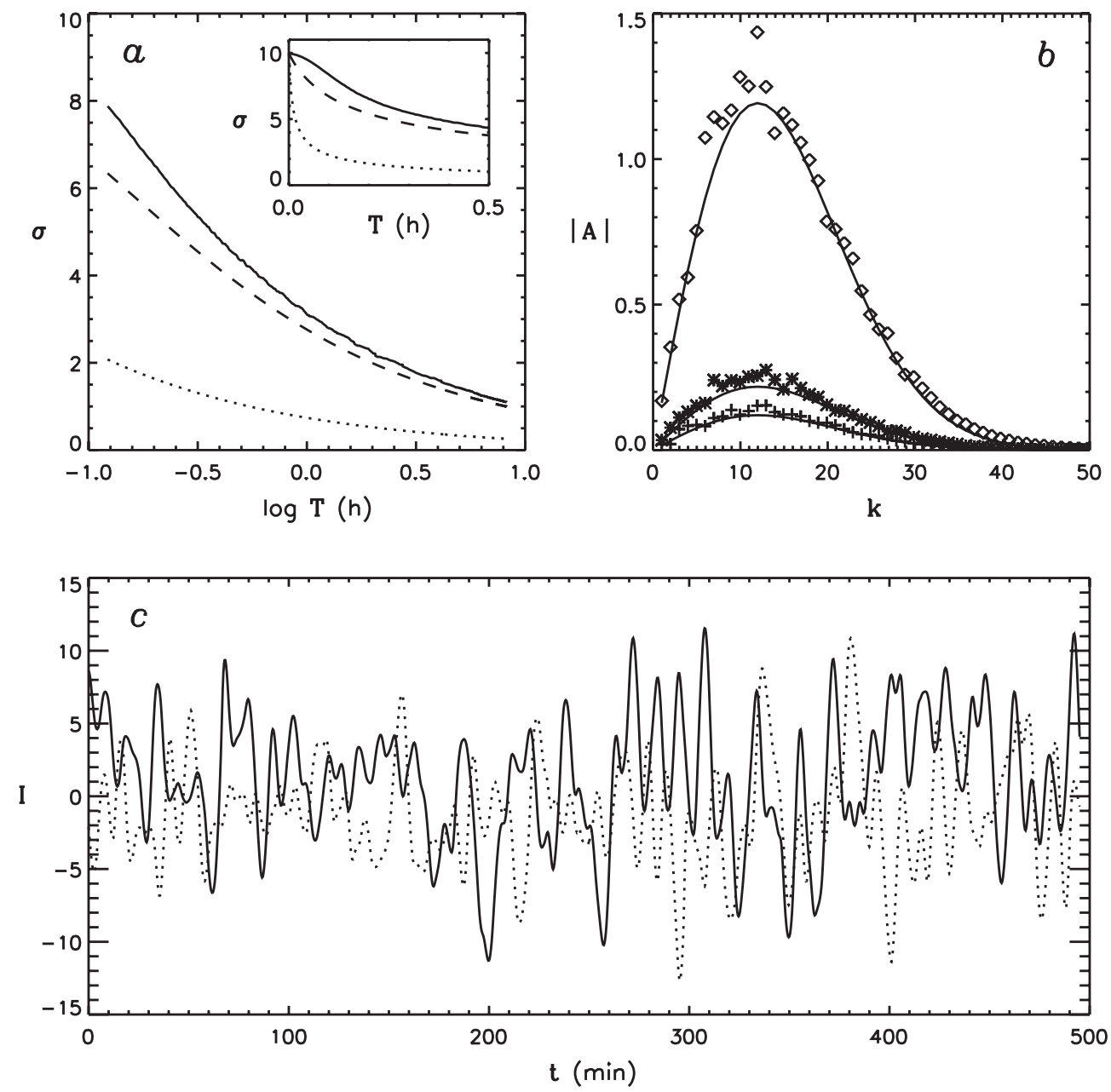

Fig. 2. In a), the root-mean-square contrast $\sigma$ of the images as a function of the averaging time $T$ (solid curve). Dotted and dashed curves plot $1 / \sqrt{N_{\mathrm{i}}}\left(N_{\mathrm{i}}\right.$ is the number of images averaged) and $1 / \sqrt{N_{\mathrm{l}}}\left(N_{\mathrm{l}}=1+T / \tau\right.$, the number of independent images averaged) respectively for comparison. Inset shows detail (on a linear-linear scale) for short averaging times. In b), the spatial amplitude spectra of the images in Figs. 1a-c (shown with diamonds, asterisks, and crosses respectively) and the expected spectra (solid curves) from Eq. (1) (with $N=N_{\mathrm{l}}$ and all Gaussians sharing the same mean width). In c), time series of the image intensity $I$ at a locally bright location (solid curve) and a nearby dark location (dashed curve) from Fig. 1c.

granulation images are consistent with those obtained from an evolving random field composed of objects with lifetimes of $5 \mathrm{~min}$. The repetition of bright or dark events at locally bright or dark locations in average images is statistically necessary to produce the fluctuations in those mean images. Large scale structures at moderate averaging times do not reflect real flows and their lifetimes, but the relatively small number statistics over those time periods and, possibly, the degradation in resolution of the sharp intergranular lanes inherent in the averaging process.
Acknowledgements. Special thanks to F. Berrilli, T. Brown, I. Ermolli, T. Holzer, A. López Ariste, and K. MacGregor.

\section{References}

Getling, A. V., \& Brandt, P. N. 2002, A\&A, 382, L5

Maunder, E. W. 1894, Knowledge, 17, 249 